OPEN ACCESS

Edited by:

Jussi Tohka,

University of Eastern Finland, Finland

Reviewed by:

Xiaohua Cao

First Hospital of Shanxi Medical

University, China

Vesa Olavi Korhonen,

Oulu University Hospital, Finland

*Correspondence:

Dongbin Li

lidb0033@163.com

Yafen Wei

weiyafensinyk@163.com

Specialty section: This article was submitted to Applied Neuroimaging, a section of the journal

Frontiers in Neurology

Received: 03 August 2021 Accepted: 27 October 2021 Published: 29 November 2021

Citation

Gao Y, Wang X, Xiong Z, Ren H, Liu R, Wei $Y$ and Li D (2021) Abnormal

Fractional Amplitude of

Low-Frequency Fluctuation as a Potential Imaging Biomarker for

First-Episode Major Depressive Disorder: A Resting-State fMRI Study and Support Vector Machine Analysis.

Front. Neurol. 12:751400. doi: 10.3389/fneur.2021.751400

\section{Abnormal Fractional Amplitude of Low-Frequency Fluctuation as a Potential Imaging Biomarker for First-Episode Major Depressive Disorder: A Resting-State fMRI Study and Support Vector Machine Analysis}

\author{
Yujun Gao ${ }^{1}$, Xi Wang ${ }^{2}$, Zhenying Xiong ${ }^{3}$, Hongwei Ren ${ }^{4}$, Ruoshi Liu ${ }^{5}$, Yafen Wei ${ }^{6 *}$ and \\ Dongbin $\mathrm{Li}^{6,7 *}$
}

\footnotetext{
${ }^{1}$ Department of Psychiatry, Renmin Hospital of Wuhan University, Wuhan, China, ${ }^{2}$ Department of Mental Health, Taihe Hospital, Hubei University of Medicine, Shiyan, China, ${ }^{3}$ Department of Psychiatry, Jiangxia District Mental Hospital, Wuhan, China, ${ }^{4}$ Department of Medical Imaging, Tianyou Hospital Affiliated to Wuhan University of Science and Technology, Wuhan, China, ${ }^{5}$ Department of Neurology, The Fourth Affiliated Hospital of Harbin Medical University, Harbin, China, ${ }^{6}$ First Department of Neurology and Neuroscience Center, Heilongjiang Provincial Hospital, Harbin, China, ${ }^{7}$ Department of Neurology, The First Affiliated Hospital of Harbin Medical University, Harbin, China
}

Objective: Major depressive disorder (MDD) is a psychiatric disorder with serious negative health outcomes; however, there is no reliable method of diagnosis. This study explored the clinical diagnostic value of the fractional amplitude of low-frequency fluctuation (fALFF) based on the support vector machine (SVM) method for the diagnosis of MDD.

Methods: A total of 198 first-episode MDD patients and 234 healthy controls were involved in this study, and all participants underwent resting-state functional magnetic resonance imaging (fMRI) scanning. Imaging data were analyzed with the fALFF and SVM methods.

Results: Compared with the healthy controls, the first-episode MDD patients showed higher fALFF in the left mid cingulum, right precuneus, and left superior frontal gyrus (SFG). The increased fALFF in these three brain regions was positively correlated with the executive control reaction time (ECRT), and the increased fALFF in the left mid cingulum and left SFG was positively correlated with the 17-item Hamilton Rating Scale for Depression (HRSD-17) scores. The SVM results showed that increased fALFF in the left mid cingulum, right precuneus, and left SFG exhibited high diagnostic accuracy of $72.92 \%$ (315/432), 71.76\% (310/432), and 73.84\% (319/432), respectively. The highest diagnostic accuracy of $76.39 \%$ (330/432) was demonstrated for the combination of increased fALFF in the right precuneus and left SFG, along with a sensitivity of $84.34 \%$ (167/198), and a specificity of $70.51 \%$ (165/234). 
Conclusion: Increased fALFF in the left mid cingulum, right precuneus, and left SFG may serve as a neuroimaging marker for first-episode MDD. The use of the increased fALFF in the right precuneus and left SFG in combination showed the best diagnostic value.

Keywords: fractional amplitude of low-frequency fluctuation, first-episode major depressive disorder, resting-state fMRI, support vector machine, imaging biomarker

\section{INTRODUCTION}

Major depressive disorder (MDD) is a severe psychiatric disorder affecting more than 264 million people around the world (1) and is characterized by cognitive and affective dysfunction. Previous studies have revealed that MDD participates in some mechanisms/pathophysiologies, such as genetics, environmental factors, neuroendocrinology, inflammation, neuroplasticity, and monoamines (2). All these molecular and cellular alterations ultimately result in brain network structure and functional network connectivity. Several studies have confirmed that MDD is relevant to specific brain circuits or networks, such as the default mode network (DMN) (3), affective-salience circuit (4), and frontoparietal cognitive control circuit (5). Concomitantly, abnormal brain circuits/networks have also been confirmed to be related to the symptoms of MDD. Frontoparietal network hypoconnectivity was found in MDD and was associated with attention regulation (6). Major depressive disorder is related to activity within the subcallosal cingulate cortex, an extensively connected element of the limbic system that regulates feelings of sadness (7). Major depressive disorder was also associated with decreased connectivity between the ventromedial prefrontal cortex and dorsal striatum, which correlated with motor speed and psychomotor slowing (8).

With the rapid development of neuroimaging technology, particularly resting-state functional magnetic resonance imaging (fMRI), our understanding of MDD mechanisms may move a step further. Due to its non-invasive characteristics, restingstate fMRI has been widely used in the neuropathology of neuropsychiatric disorders, such as autism (9), obsessivecompulsive disorder (10), anxiety disorders (11), and MMD (12). Resting-state fMRI relies on the spontaneous low-frequency fluctuation in the blood oxygen level-dependent signal, which could reflect the spontaneous activity of neurons (13). During resting-state fMRI, subjects were asked to be awake and quiet without conscious mental activity. Compared with taskstate fMRI, resting-state fMRI is more convenient for clinical application. Several analysis methods of resting-state fMRI have been widely used in the assessment of disease processes and disease diagnostics, such as regional homogeneity (14), voxel-based morphometry (15), network homogeneity (16), voxel-mirrored homotopic (17), functional connectivity (18), and mean-square successive difference (19). Although most resting-state $\mathrm{fMRI}$ studies have confirmed correlations between spatially different brain regions from functional integration, these methods do not intuitively provide information on the amplitude of brain activity in all brain regions. For instance, regional homogeneity is employed mainly in the measurement of the functional coherence of a given voxel with its nearest voxels (20), and functional connectivity is used mainly for measuring connections between brain areas or individual voxels within a network $(21,22)$.

The fractional amplitude of low-frequency fluctuation (fALFF) is frequently used for the brain function evaluation and can be used to characterize spontaneous brain activity (23). Due to the high temporal stability, fALFF was confirmed to have high potential value as a diagnostic marker in a previous study (24). Compared with amplitude of low-frequency fluctuation (ALFF) analysis (the original approach), fALFF has fewer physiological noise effects (25). Although the number of MDD studies using fALFF is increasing; few studies have reported the combination of fALFF and support vector machine (SVM) methods. The SVM method is a multivariable pattern recognition technology that can be used to analyze the data and detect patterns (26). Meanwhile, the SVM is ideally suited for high-dimensional data, with many more features than the number of samples, which is often true of experiments. An optimal separating hyperplane of the high-dimensional space can be confirmed via the SVM, and the samples that are closest to the hyperplane are called the support vector. In the fMRI analysis, a discrimination map can be generated by superimposing the SVM weights back onto the original brain space, so the most significant weights can be visually traced back to the most discriminatory parts of the brain (27). The SVM method has great potential to provide clinically useful criteria for decision-making from such high-dimensional neuroimaging data (28). In this study, we investigated fALFF in first-episode MDD patients, screened brain regions that showed altered fALFF, and discussed their value as potential neuroimaging markers through the SVM method. This study will contribute to the rapid and efficient diagnosis of first-episode MDD.

\section{METHODS}

\section{Subjects}

One hundred ninety-eight right-handed first-episode MDD patients were consecutively recruited from the Department of Psychiatry, Tianyou Hospital Affiliated to Wuhan University of Science and Technology. Two hundred thirty-four age-matched (MDD patients: $28.01 \pm 7.442$, healthy controls: $27.87 \pm 6.492$ ) and gender-matched (MDD patients: men/women $=102 / 96$, healthy controls: 130/104) right-handed healthy controls were recruited from those who underwent a standard physical 
examination at the medical examination center of the Tianyou Hospital Affiliated to Wuhan University of Science and Technology. The diagnosis of depression was based on the Diagnostic and Statistical Manual of Mental Disorders-Fourth Edition (DSM-IV) criteria (29), and all the patients were independently diagnosed by two experienced psychiatrists. Depression severity was assessed by the 17-item Hamilton Rating Scale for Depression (HRSD-17) score, and the patients had a score of more than 18. In the process of clinician-rated depression, the application of HRSD-17 was most prevalent. Based on the HRSD-17, the severity of depression was divided into four categories: $<7$ scores $=$ normal people, $8-16$ scores $=$ mild depression, $17-23$ scores $=$ moderate depression, and $>24$ scores $=$ severe depression. The exclusion criteria for patients were as follows: other Axis I disorders, such as bipolar disorder, acute physical illness, schizophrenia, substance abuse or dependence, substance-induced mood disorder, and a history of head injury resulting in the loss of consciousness. The healthy controls had no history of psychiatric disorders or severe physical illness and no family history of psychiatric disorders. All participants gave written informed consent before the study. Our study was approved by the Medical Ethics Committee of the Tianyou Hospital Affiliated to Wuhan University of Science and Technology and performed in accordance with the Declaration of Helsinki.

\section{Behavioral Paradigms}

The executive control function was assessed by the attentional network test (ANT) (30). The stimulus signals of ANT visually appear on a screen, and the subjects were required to correctly and quickly identify the orientation in which a central target arrow pointed. The reaction time (RT) of all the subjects was recorded, and the executive control reaction time (ECRT) was calculated by subtracting the consistent arrow direction RT from the inconsistent arrow direction RT. A longer ECRT indicated inferior executive control performance [the detailed steps were excerpted from our previous study (31), and detailed information can be found in the Supplementary Material].

\section{Image Acquisition}

All the resting-state MRI data were acquired on an Achieva 3.0T scanner (Philips, Amsterdam, the Netherlands) at the Tianyou Hospital Affiliated to Wuhan University of Science and Technology. All the participants were instructed to lie still and stay awake with their eyes closed. The scanning parameters were as follows: repetition time/echo time (TR/TE) 2,000/30 ms; 31 slices; $90^{\circ}$ flip angle; $220 \times 220 \mathrm{~mm}$ field of view; $5 \mathrm{~mm}$ slice thickness; and $1 \mathrm{~mm}$ pitch [the detailed steps were excerpted from our previous study (32), and detailed information can be found in the Supplementary Material].

\section{Data Preprocessing}

Imaging data from the resting-state $\mathrm{AMRI}$ were preprocessed using the DPARSF software in MATLAB (23). To reduce the influence of participants' adaption time and the instability of the initial signal, the first five-time points were discarded. Slice time and head motion were corrected. None of the participants had more than $2 \mathrm{~mm}$ of maximum displacement in the $\mathrm{x}^{-}, \mathrm{y}^{-}$, or $\mathrm{z}$-axis, or more than $2^{\circ}$ of maximum rotation. The corrected imaging data were spatially normalized to the standard Montreal Neurological Institute space and resampled with $1 \times 1 \times 1 \mathrm{~mm}^{3}$. Then, the obtained images were band-pass filtered $(0.01-0.08 \mathrm{~Hz})$ and linearly detrended. Several spurious covariates were removed, such as the signal from a region centered in the white matter and the signal from a ventricular seed-based region of interest, as well as the six head-motion parameters obtained by rigid body correction. The global signal was retained during the processing of the resting-state functional connectivity data [the detailed steps were excerpted from our previous study (32), and detailed information can be found in the Supplementary Material].

\section{fALFF Analysis}

The fALFF analysis was performed with REST software. The fALFF values were calculated according to the previous study (33). The time series for each voxel were transformed to the frequency domain by using fast Fourier transform, and the power spectrum was then computed and square roottransformed at each voxel. The averaged square root was obtained as the ALFF across $0.01-0.08 \mathrm{~Hz}$ at each voxel. After that ALFF was calculated, the sum of amplitudes across $0.01-0.08 \mathrm{~Hz}$ was divided by that across the entire frequency range, and fALFF was acquired (detailed information can be found in the Supplementary Material).

\section{Classification Analysis}

The SVM method was operated using the LIBSVM software package in MATLAB and was applied to test the ability to differentiate MDD from healthy controls using the extracted fALFF values in abnormal brain regions. The best parameters, $\mathrm{C}$ (penalty coefficient), and g (gamma) were selected, and the LIBSVM tool was used to evaluate a grid of parameters. Through the LIBSVM, all the parameter settings' accuracies were acquired, and then, the highest cross-validation accuracy of the parameters was determined (detailed information can be found in the Supplementary Material).

\section{Statistics}

The two-sample $t$-test was used to analyze the age, years of education, HRSD-17, and ECRT between MDD patients and

TABLE 1 | The $p$-value for the gender distribution was obtained by the Chi-square test.

\begin{tabular}{lccc}
\hline Characteristics & Patients $(\boldsymbol{n}=\mathbf{1 9 8})$ & HCs $(\boldsymbol{n}=\mathbf{2 3 4})$ & $\boldsymbol{p}$-values \\
\hline Gender (men/women) & $198(102 / 96)$ & $234(130 / 104)$ & 0.401 \\
Age, years & $28.01 \pm 7.442$ & $27.87 \pm 6.492$ & 0.832 \\
Years of education, years & $12.05 \pm 3.325$ & $12.55 \pm 2.931$ & 0.100 \\
HRSD-17 & $23.63 \pm 2.547$ & & \\
ECRT (ms) & $141.10 \pm 49.400$ & $84.11 \pm 52.746$ & 0.000
\end{tabular}

The other p-values were obtained by two sample t-tests. HC, healthy controls; ECRT, executive control reaction time; HRSD-17, 17-item Hamilton rating scale for depression. 
healthy controls, and the Chi-squared test was used to analyze the gender distributions using SPSS 22.0. Age, years of education, and frame-wise displacement all were used as covariates. Spearman correlation was used to detect any correlations between abnormal fALFF and clinical variables. The significance level was set at $p<0.05$.

To identify the group differences, an analysis of covariance was used on individual whole-brain fALFF maps of the two groups in a voxel-by-voxel manner. The voxel-based mean fALFF values were withdrawn from the abnormal values in brain regions. The results were thresholded at $p<0.01$, GRF-corrected using cluster-extent based thresholding in REST with primary threshold of $p<0.001$.

\section{RESULTS}

\section{Characteristics of the Subjects}

A total of 198 first-episode MDD patients and 234 healthy controls were involved in this research. The demographic and clinical data of the participants are provided in Table 1. No significant differences were observed in terms of age, sex, or educational level. However, first-episode MDD patients had a longer ECRT than healthy controls.

\section{Group Differences in fALFF}

The two-sample $t$-test was used to test significant differences in fALFF values between first-episode MDD patients and healthy controls. Patients showed significantly higher fALFF in the left mid cingulum, right precuneus, and left superior frontal gyrus (SFG) than healthy controls (Figure 1; Table 2).

\section{SVM Results}

The increased fALFF values of these three brain regions (left mid cingulum, right precuneus, and left SFG) in the firstepisode MDD patients were analyzed by the SVM method. The classification accuracies were as follows: left mid cingulum = $72.9167 \%$ (315/432), right precuneus $=71.7593 \%(310 / 432)$, and left SFG $=73.8426 \%(319 / 432)$. The increased fALFF in the left SFG showed the highest diagnostic accuracy of $73.8426 \%$, with a sensitivity of $81.82 \%(162 / 198)$, and a specificity of $72.65 \%$ (170/234) (Figure 2). Meanwhile, the SVM results showed that a combination of increased fALFF in the right precuneus and left SFG exhibited the highest accuracy of $76.3889 \%$ (330/432), with a sensitivity of $84.34 \%(167 / 198)$, and a specificity of $70.51 \%$ (165/234) (Figure 3).

\section{Correlation of fALFF With Clinical Variables}

As shown in Figure 4, increased fALFF values in the left mid cingulum, right precuneus, and left SFG were positively correlated with ECRT. The increased fALFF values in the left

TABLE 2 | Clusters with abnormal fractional amplitude of low-frequency fluctuation in the patients with major depressive disorder.

\begin{tabular}{|c|c|c|c|c|c|}
\hline \multirow[t]{2}{*}{ Cluster location } & \multicolumn{3}{|c|}{ Peak (MNI) } & \multirow[t]{2}{*}{ Number of voxels } & \multirow[t]{2}{*}{$T$-value } \\
\hline & $x$ & $Y$ & $Z$ & & \\
\hline Left mid cingulum & -24 & -27 & 30 & 145 & 8.0602 \\
\hline Right precuneus & 15 & -48 & 21 & 235 & 8.5775 \\
\hline Left SFG & -21 & 24 & 24 & 58 & 8.0042 \\
\hline
\end{tabular}

MNI, Montreal neurological institute; SFG, superior frontal gyrus.
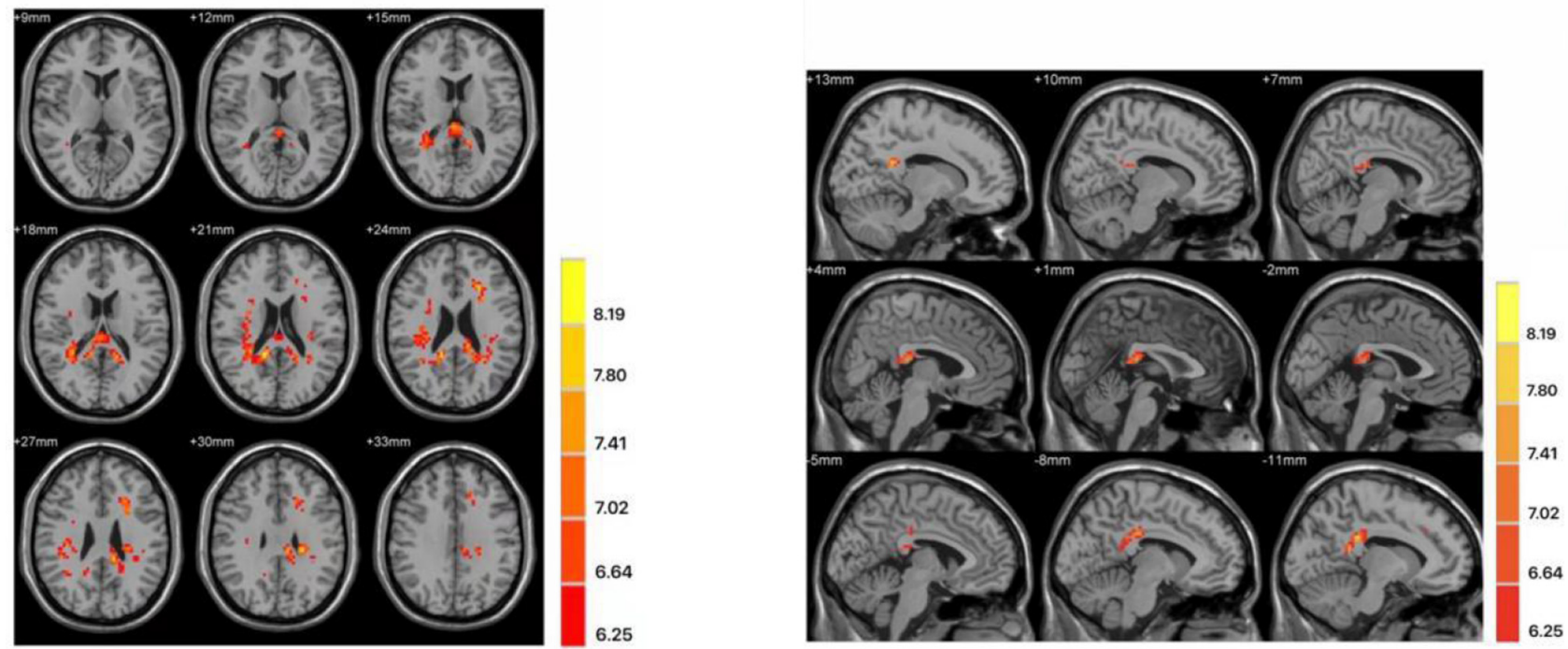

FIGURE 1 | Differences in fractional amplitude of low-frequency fluctuation (fALFF) values between first-episode major depressive disorder patients and healthy controls. Increased fALFF values (left mid cingulum, right precuneus, and left superior frontal gyrus) were presented on the red color, and the color bar indicates the $T$ values of the group analysis. Left: transverse plane; Right: sagittal plane. 

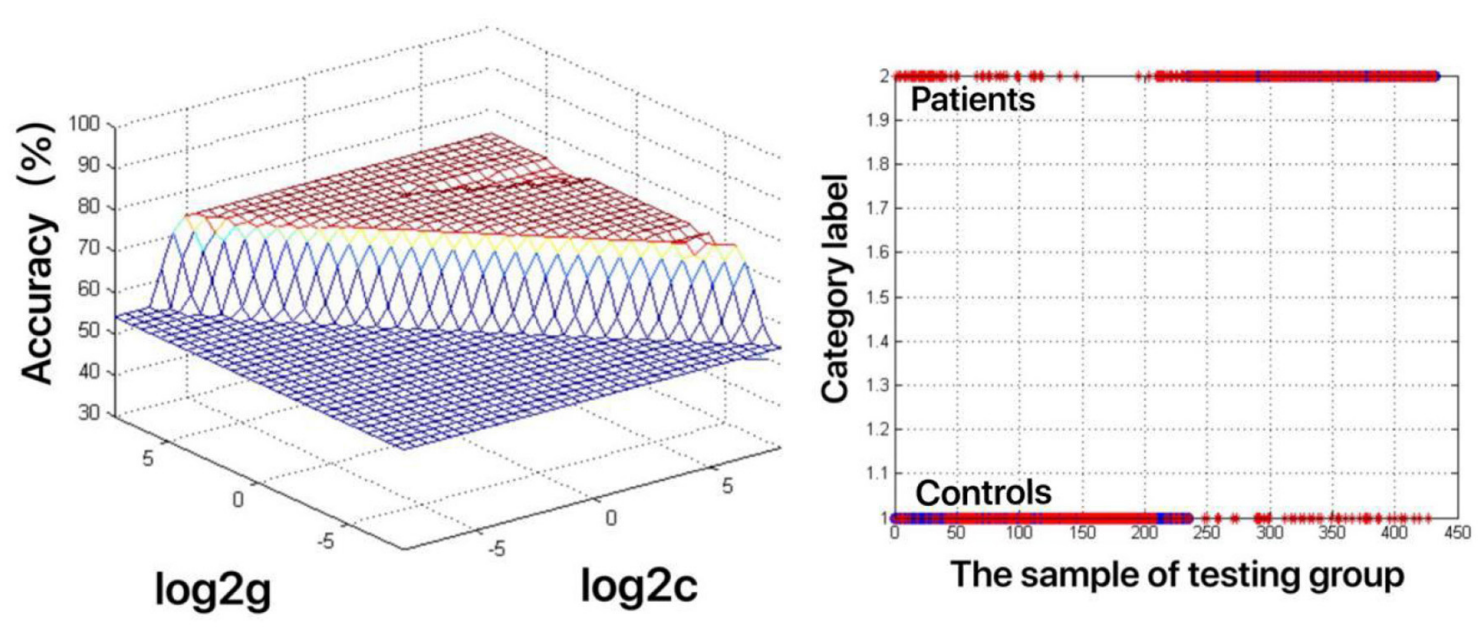

FIGURE 2 | Visualization of classifications through support vector machine (SVM) using the increased fractional amplitude of low-frequency fluctuation (fALFF) values in the left superior frontal gyrus (SFG) to discriminate the MDD patients from healthy controls. Left: SVM parameters result of 3D view. $g$ means gamma, c means penalty coefficient. Right: Classified map of the fALFF values in the left SFG. Blue circle means true value and the red asterisk means predict value.
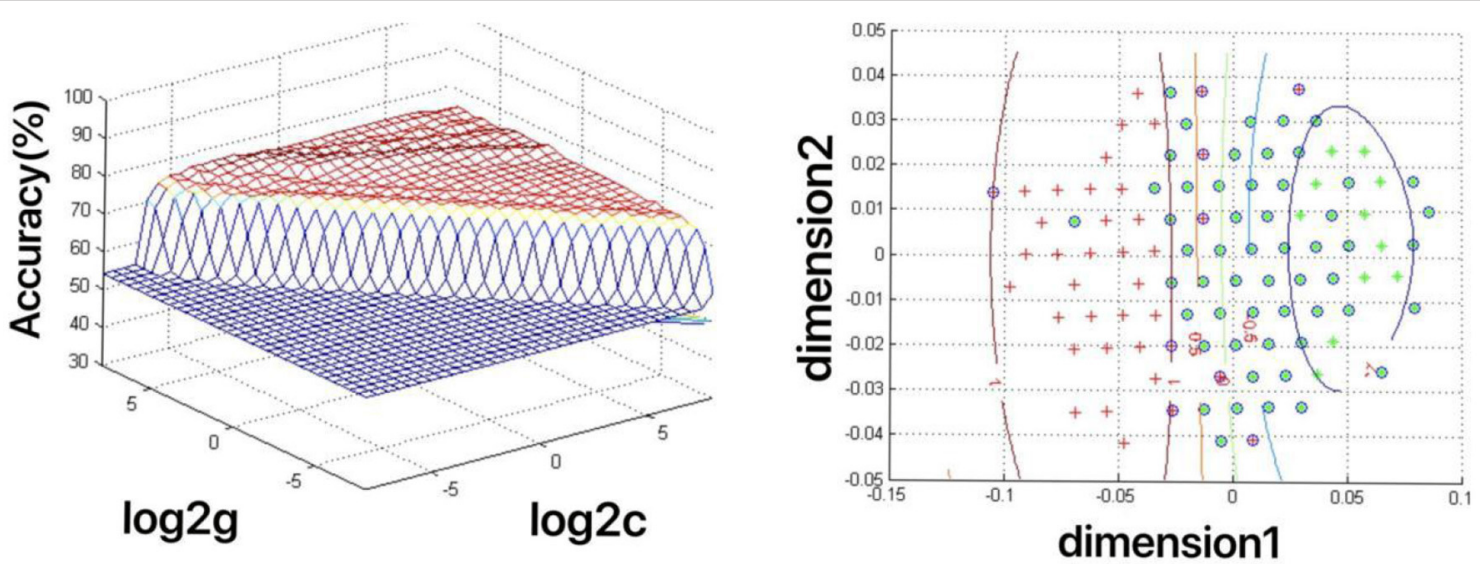

FIGURE 3 | Depiction of classifications based on the support vector machine (SVM) method using a combination of fractional amplitude of low-frequency fluctuation ( $f$ ALFF) values in the right precuneus and left SFG to differentiate the first-episode major depressive disorder (MDD) patients from the healthy controls. Left: SVM parameters result of 3D view. g means gamma, c means penalty coefficient. Right: dimension 1 and dimension 2 represent the fALFF values in the right precuneus and left SFG, respectively. Green crosses represent the first-episode MDD patients, and the red crosses represent the healthy controls.

mid cingulum and left SFG were positively correlated with the HRSD-17 scores (Figure 5).

\section{DISCUSSION}

The objective and rapid diagnosis of MDD have always been a hot spot in clinical research; however, there are no objective lab-based diagnostic methods for MDD, and its diagnosis still depends on depression scales and subjective analysis. With the development of brain imaging technology, fMRI research is increasingly used to assist clinical diagnosis. To the best of our knowledge, this study was the first to explore the utility of altered fALFF values in the left mid-cingulum, right precuneus, and left SFG as neuroimaging markers for the first-episode MDD, combined with the SVM method.

The cingulum is a key component of the limbic lobe, and it is a major interconnecting apparatus of all cerebral lobes (34). It has been described as the "seat of dynamic vigilance by which environmental experiences are endowed with an emotional awareness" by Papez (35). Due to its cytoarchitectonic characteristics and the distribution of receptors, the cingulate gyrus is divided into four subregions, such as the anterior cingulate cortex, middle cingulate cortex, posterior cingulate cortex, and retrosplenial cortex (36). The middle cingulate cortex has been implicated in negative affect and cognitive control (37) and is mainly enrolled in the selection of responses according to their motivational relevance (34). Moreover, several memory 

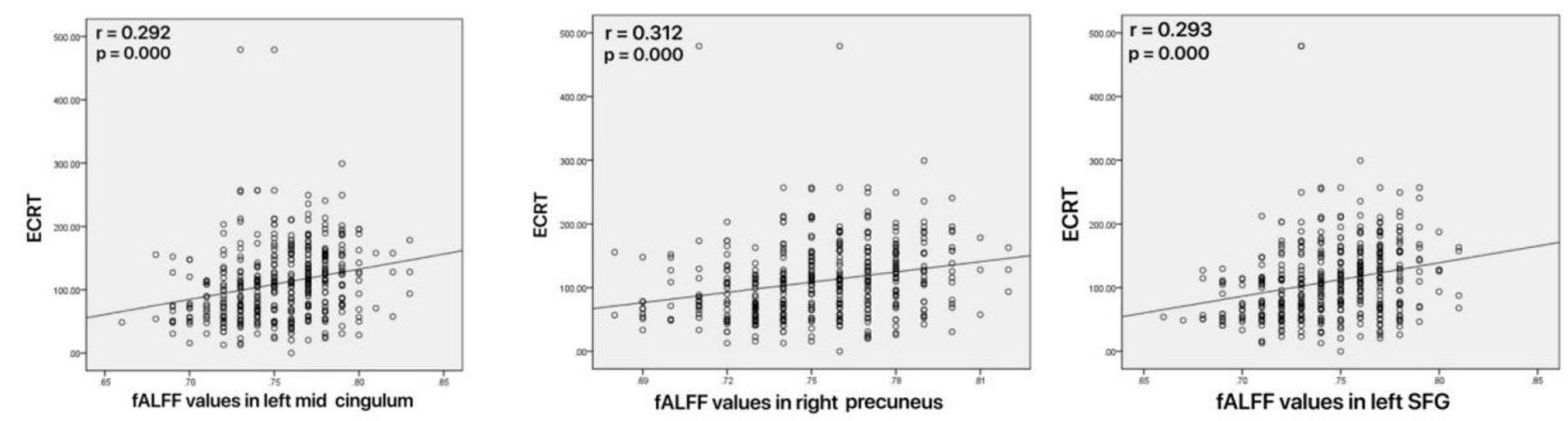

FIGURE 4 | Correlations between abnormal fractional amplitude of low-frequency fluctuation (fALFF) and executive control reaction time (ECRT). Left: Positive correlation between the fALFF values in the left mid cingulum and ECRT; Mid: Positive correlation between the fALFF values in the right precuneus and ECRT; Right: Positive correlation between the fALFF values in the left SFG and ECRT.

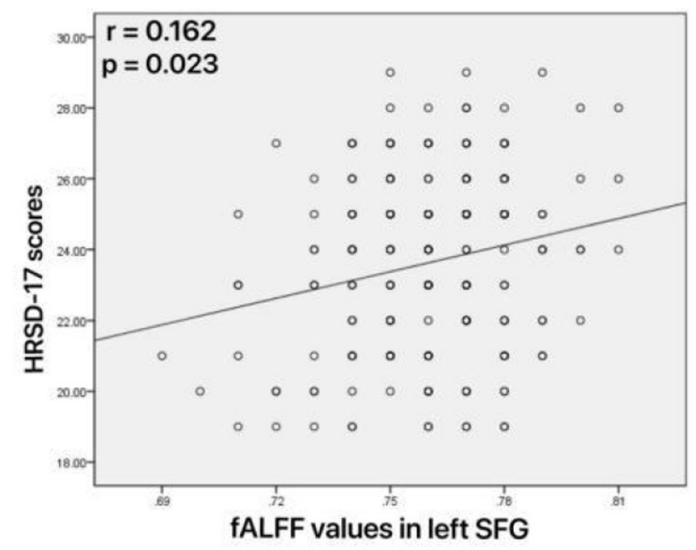

FIGURE 5 | Correlations between abnormal fractional amplitude of low-frequency fluctuation (fALFF) and 17-item Hamilton Rating Scale for Depression (HRSD-17) scores. Positive correlation between the fALFF values in the left superior frontal gyrus and HRSD-17 scores.

task studies have shown evidence that the cingulum participates in working memory (38), especially the mid cingulum. Although the memory task was not included in the present study, similar results were noticed. In our study, an ANT was performed to evaluate executive control function, and first-episode MDD patients showed longer ECRT than healthy controls. Consistent with our study, Rao et al. (39) confirmed that the left mid cingulum is closely related to attention networks, which also modulate cognitive-linguistic conflict. Cerebral perfusion singlephoton emission computed tomography (40) revealed that the number of depressive episodes was negatively correlated with perfusion of the right anterior cingulum, and the depression duration was negatively correlated with perfusion of the right anterior cingulum. In our study, increased fALFF values of the left mid cingulum were found in the patients with MDD, and the altered fALFF values were positively correlated with ECRT. As mentioned above, a longer ECRT indicated inferior executive control performance, and thus we speculated that abnormal fALFF in the left mid cingulum has a critical role in the executive function of MDD.

The precuneus is one of the highest resting metabolic rates of all brain structures and is located on the posteromedial portion of the parietal lobe. As the precuneus is hidden in the interhemispheric fissure and rarely damaged in brain diseases such as ischemic stroke and accidents, this cortical area has traditionally received little study. The precuneus, as the functional core of the DMN, was one of the most important findings of recent neuroimaging studies $(41,42)$. However, the precuneus is closely related to the DMN, which is thought to be tightly associated with MDD, we confirmed in our present study that the precuneus is involved in the process of MDD. Hermesdorf et al. (43) found that compared with never-depressed controls, MDD patients showed lower voxelmirrored homotopic connectivity (VMHC) in the precuneus. In the present study, we observed increased fALFF values in the right precuneus, and the SVM results showed an accuracy of $71.7593 \%$ (310/432) to differentiate the first-episode MDD from healthy controls. Although no correlation between fALFF and HRSD-17 scores was found in our study, the increased fALFF of the right precuneus was positively correlated with ECRT.

The SFG is an important functional region located in the superior prefrontal cortex. Based on diffusion tensor tractography, the SFG can be divided into three subregions: the anteromedial SFG, dorsolateral SFG, and posterior SFG (44). Remarkably, both the anteromedial SFG and dorsolateral SFG were mainly correlated with the DMN (44), similar to the precuneus. Meanwhile, the SFG also plays a critical role in working memory, similar to the cingulum $(45,46)$. From all the descriptions discussed above, it is not difficult to find that there is a tight functional correlation among these three brain regions (precuneus, cingulum, and SFG) in our study. Several studies on the abnormal SFG in MDD have been reported; for example, Xiong et al. (47) confirmed that altered left SFG gyrification means state-independent changes in MDD. Lebedeva et al. (48) found that atrophy in the left SFG developed in parallel with depressive symptoms. In the present study, we observed 
increased fALFF values in the left SFG, and the SVM results showed an accuracy of $73.8426 \%(319 / 432)$ to differentiate the first-episode MDD from healthy controls. However, a previous study showed the opposite result, which suggested that decreased fALFF in the left SFG may be a trait neuroimaging marker for MDD (49). We hypothesized that the number of samples was the key factor (only $14 \mathrm{MDD}$ patients were enrolled in the previous study). Our results showed increased fALFF values in the left SFG, and the altered fALFF values were positively correlated with the ECRT and HRSD-17 scores. Thus, our data suggested that increased fALFF of the left SFG may contribute to the pathophysiological processing of MDD.

Over recent years, neuroimaging markers have become an intense focus of MDD diagnosis. Many neuroimaging studies have been reported, such as brain volumetric MRI (50), diffusion tensor imaging (51), and single-photon emission computed tomography (52). However, the results of these studies are inconsistent, and several factors, such as different study designs and small sample sizes, may contribute to this inconsistency. In this study, we collected a large sample size and chose the SVM method, which has been widely used for computeraided diagnosis and prediction in disease diagnosis. Fractional amplitude of low-frequency fluctuation is a common index of resting-state fMRI, which is closely associated with the pathophysiological mechanisms of MDD. By measuring the abnormal fALFF values of MDD and first-degree relatives, Song et al. (53) suggested that fALFF could be utilized as a neuroimaging marker for monitoring MDD progression. Qiu et al. (54) reported that after electroconvulsive therapy, MDD patients showed obviously decreased fALFF. In our study, SVM analysis showed that increased fALFF values in the left mid cingulum, right precuneus, and left SFG could be used to distinguish first-episode MDD patients from healthy controls, and the combination of increased fALFF in the right precuneus and left SFG exhibited the highest accuracy of $76.3889 \%$ (330/432), with a sensitivity of $84.34 \%(167 / 198)$, and a specificity of $70.51 \%(165 / 234)$.

Some limitations exist in our study. The influence of physiological noises such as cardiac and respiratory rhythm cannot be completely removed. Another limitation is the sample source as all the subjects were recruited from the China's Hubei province.

\section{REFERENCES}

1. Duan L, Gao Y, Shao X, Tian C, Fu C, Zhu G. Research on the development of theme trends and changes of knowledge structures of drug therapy studies on major depressive disorder since the 21 st century: a bibliometric analysis. Front Psychiatry. (2020) 11:647. doi: 10.3389/fpsyt.2020. 00647

2. Otte C, Gold SM, Penninx BW, Pariante CM, Etkin A, Fava M, et al. Major depressive disorder. Nature Rev Dis Primers. (2016) 2:16065. doi: 10.1038/nrdp.2016.65

3. Scalabrini A, Vai B, Poletti S, Damiani S, Mucci C, Colombo C, et al. All roads lead to the default-mode network-global source of DMN abnormalities in major depressive disorder. Neuropsychopharmacology. (2020) 45:205869. doi: 10.1038/s41386-020-0785-x

\section{CONCLUSION}

In conclusion, the altered fALFF in the left mid cingulum, right precuneus, and left SFG may be state-related changes of MDD. And, the combination of increased fALFF in the right precuneus and left SFG may be a potential neuroimaging marker for the first-episode MDD.

\section{DATA AVAILABILITY STATEMENT}

The raw data supporting the conclusions of this article will be made available by the authors, without undue reservation.

\section{ETHICS STATEMENT}

The studies involving human participants were reviewed and approved by the Medical Ethics Committee of the Tianyou Hospital Affiliated to Wuhan University of Science and Technology. The patients/participants provided their written informed consent to participate in this study. Written informed consent was obtained from the individual(s) for the publication of any potentially identifiable images or data included in this article.

\section{AUTHOR CONTRIBUTIONS}

DL and RL: writing the article. XW, ZX, and HR: collection and assembly of data. YG and YW: research concept and design, data analysis, and interpretation. All authors contributed to the article and approved the submitted version.

\section{FUNDING}

The investigation was supported by the grant from the Health Commission of Hubei Province Scientific research project (Grant No. 2020CFB512).

\section{SUPPLEMENTARY MATERIAL}

The Supplementary Material for this article can be found online at: https://www.frontiersin.org/articles/10.3389/fneur. 2021.751400/full\#supplementary-material

4. Hamilton JP, Etkin A, Furman DJ, Lemus MG, Johnson RF, Gotlib IH. Functional neuroimaging of major depressive disorder: a meta-analysis and new integration of base line activation and neural response data. Am J Psychiatry. (2012) 169:693-703. doi: 10.1176/appi.ajp.2012.11071105

5. Zhang Y, Wu W, Toll RT, Naparstek S, Maron-Katz A, Watts M, et al. Identification of psychiatric disorder subtypes from functional connectivity patterns in resting-state electroencephalography. Nat Biomed Eng. (2021) 5:309-23. doi: 10.1038/s41551-020-00614-8

6. Kaiser RH, Andrews-Hanna JR, Wager TD, Pizzagalli DA. Large-scale network dysfunction in major depressive disorder: a meta-analysis of resting-state functional connectivity. JAMA psychiatry. (2015) 72:60311. doi: 10.1001/jamapsychiatry.2015.0071

7. Smith R, Fadok RA, Purcell M, Liu S, Stonnington C, Spetzler RF, et al. Localizing sadness activation within the subgenual cingulate in individuals: 
a novel functional MRI paradigm for detecting individual differences in the neural circuitry underlying depression. Brain Imaging Behav. (2011) 5:22939. doi: 10.1007/s11682-011-9127-2

8. Felger JC, Li Z, Haroon E, Woolwine BJ, Jung MY, Hu X, et al. Inflammation is associated with decreased functional connectivity within corticostriatal reward circuitry in depression. Mol Psychiatry. (2016) 21:135865. doi: $10.1038 / \mathrm{mp} .2015 .168$

9. Mash LE, Keehn B, Linke AC, Liu TT, Helm JL, Haist F, et al. Atypical relationships between spontaneous EEG and fMRI activity in autism. Brain Connect. (2020) 10:18-28. doi: 10.1089/brain.2019.0693

10. Viol K, Aas B, Kastinger A, Kronbichler M, Schöller H, Reiter EM, et al. Individual OCD-provoking stimuli activate disorder-related and self-related neuronal networks in fMRI. Psychiatry Res Neuroimaging. (2019) 283:13544. doi: 10.1016/j.pscychresns.2018.12.008

11. Gorka SM, Young CB, Klumpp H, Kennedy AE, Francis J, Ajilore O, et al. Emotion-based brain mechanisms and predictors for SSRI and CBT treatment of anxiety and depression: a randomized trial. Neuropsychopharmacology. (2019) 44:1639-48. doi: 10.1038/s41386-019-0407-7

12. Korgaonkar MS, Grieve SM, Etkin A, Koslow SH, Williams LM. Using standardized fMRI protocols to identify patterns of prefrontal circuit dysregulation that are common and specific to cognitive and emotional tasks in major depressive disorder: first wave results from the iSPOT-D study. Neuropsychopharmacology. (2013) 38:863-71. doi: 10.1038/npp.2012.252

13. Matthews PM, Jezzard P. Functional magnetic resonance imaging. J Neurol Neurosurg Psychiatry. (2004) 75:6-12.

14. Shukla DK, Keehn B, Müller RA. Regional homogeneity of fMRI time series in autism spectrum disorders. Neurosci Lett. (2010) 476:4651. doi: 10.1016/j.neulet.2010.03.080

15. Nemoto K. Understanding voxel-based morphometry. Brain Nerve. (2017) 69:505-11. doi: 10.11477/mf.1416200776

16. Respino M, Hoptman MJ, Victoria LW, Alexopoulos GS, Solomonov N, Stein AT, et al. Cognitive control network homogeneity and executive functions in late-life depression. Biol Psychiatry Cogn Neurosci Neuroimaging. (2020) 5:213-21. doi: 10.1016/j.bpsc.2019.10.013

17. Wei J, Wei S, Yang R, Yang L, Yin Q, Li H, et al. Voxel-mirrored homotopic connectivity of resting-state functional magnetic resonance imaging in blepharospasm. Front Psychol. (2018) 9:1620. doi: 10.3389/fpsyg.2018.01620

18. Rizkallah J, Amoud H, Fraschini M, Wendling F, Hassan M. Exploring the correlation between M/EEG source-space and fMRI networks at rest. Brain Topogr. (2020) 33:151-60. doi: 10.1007/s10548-020-00753-w

19. Seidel M, Geisler D, Borchardt V, King JA, Bernardoni F, Jaite C, et al. Evaluation of spontaneous regional brain activity in weight-recovered anorexia nervosa. Transl Psychiatry. (2020) 10:395. doi: 10.1038/s41398-020-01081-0

20. Liu Y, Wang K, Yu C, He Y, Zhou Y, Liang M, et al. Regional homogeneity, functional connectivity and imaging markers of Alzheimer's disease: a review of resting-state fMRI studies. Neuropsychologia. (2008) 46:164856. doi: 10.1016/j.neuropsychologia.2008.01.027

21. Paul S, Sharfman N. Functional connectivity as a means to delineate differences between treatment-resistant and treatment-responsive schizophrenia. J Neurophysiol. (2016) 116:229-31. doi: 10.1152/jn.011 27.2015

22. Martynova O, Balaev V, Sushiskaia-Tetereva A, Ivanitsky A. Correlation of resting state function connectivity with behavioral and psychological indices. Zh Vyssh Nerv Deiat Im I P Pavlova. (2016) 66:541-55.

23. Wang L, Li X, Li K, Su Y, Zeng Y, Zhang Q, et al. Mapping the effect of escitalopram treatment on amplitude of low-frequency fluctuations in patients with depression: a resting-state fMRI study. Metab Brain Dis. (2017) 32:147-54. doi: 10.1007/s11011-016-9871-5

24. Küblböck M, Woletz M, Höflich A, Sladky R, Kranz GS, Hoffmann A, et al. Stability of low-frequency fluctuation amplitudes in prolonged resting-state fMRI. Neuroimage. (2014) 103:249-57. doi: 10.1016/j.neuroimage.2014.09.038

25. Zou QH, Zhu CZ, Yang Y, Zuo XN, Long XY, Cao QJ, et al. An improved approach to detection of amplitude of low-frequency fluctuation (ALFF) for resting-state fMRI: fractional ALFF. J Neurosci Methods. (2008) 172:13741. doi: 10.1016/j.jneumeth.2008.04.0122
26. Wang X, Jiao D, Zhang X, Lin X. Altered degree centrality in childhood absence epilepsy: a resting-state fMRI study. J Neurol Sci. (2017) 373:2749. doi: 10.1016/j.jns.2016.12.054

27. Shah YS, Hernandez-Garcia L, Jahanian H, Peltier SJ. Support vector machine classification of arterial volume-weighted arterial spin tagging images. Brain Behav. (2016) 6:e00549. doi: 10.1002/brb3.549

28. Gaonkar BT, Shinohara R, Davatzikos C, Alzheimers Disease Neuroimaging Initiative. Interpreting support vector machine models for multivariate group wise analysis in neuroimaging. Med Image Anal. (2015) 24:190204. doi: 10.1016/j.media.2015.06.008

29. Lobbestael J, Leurgans M, Arntz A. Inter-rater reliability of the structured clinical interview for DSM-IV axis I disorders (SCID I) and axis II disorders (SCID II). Clin Psychol Psychother. (2011) 18:75-9. doi: 10.1002/cpp.693

30. Fan J, McCandliss BD, Sommer T, Raz A, Posner MI. Testing the efficiency and independence of attentional networks. J Cogn Neurosci. (2002) 14:3407. doi: $10.1162 / 089892902317361886$

31. Gao Y, Wang M, Yu R, Li Y, Yang Y, Cui X, et al. Abnormal default mode network homogeneity in treatment-naive patients with first-episode depression. Front Psychiatry. (2018) 9:697. doi: 10.3389/fpsyt.2018.00697

32. Gao Y, Zheng J, Li Y, Guo D, Wang M, Cui X, et al. Abnormal defaultmode network homogeneity in patients with temporal lobe epilepsy. Medicine. (2018) 97:e11239. doi: 10.1097/MD.0000000000011239

33. Zou QH, Zhu CZ, Yang Y, Zuo XN, Long XY, Cao QJ, et al. An improved approach to detection of amplitude of low-frequency fluctuation (ALFF) for resting-state fMRI: fractional ALFF. J Neurosci Methods. (2008) 172:13741. doi: 10.1016/j.jneumeth.2008.04.012

34. Maldonado IL, Parente de Matos V, Castro Cuesta TA, Herbet G, Destrieux C. The human cingulum: from the limbic tract to the connectionist paradigm. Neuropsychologia. (2020) 144:107487. doi: 10.1016/j.neuropsychologia.2020.107487

35. Papez JW. A proposed mechanism of emotion. 1937. J Neuropsychiatry Clin Neurosci. (1995) 7:103-12. doi: 10.1176/jnp.7.1.103

36. Vogt BA. Pain and emotion interactions in subregions of the cingulate gyrus. Nat Rev Neurosci. (2005) 6:533-44. doi: 10.1038/nrn1704

37. Tolomeo S, Christmas D, Jentzsch I, Johnston B, Sprengelmeyer R, Matthews $\mathrm{K}$, et al. A causal role for the anterior mid-cingulate cortex in negative affect and cognitive control. Brain. (2016) 139:1844-54. doi: 10.1093/brain/aww069

38. Heany SJ, Phillips N, Brooks S, Fouche JP, Myer L, Zar H, et al. Neural correlates of maintenance working memory, as well as relevant structural qualities, are associated with earlier antiretroviral treatment initiation in vertically transmitted HIV. J Neurovirol. (2020) 26:609. doi: 10.1007/s13365-019-00792-5

39. Rao C, Mathur A, Singh NC. 'Cost in transliteration': the neurocognitive processing of Romanized writing. Brain Lang. (2013) 124:205-12. doi: 10.1016/j.bandl.2012.12.004

40. Tastevin M, Boyer L, Korchia T, Fond G, Lançon C, Richieri R, et al. Brain SPECT perfusion and PET metabolism as discordant biomarkers in major depressive disorder. EJNMMI Res. (2020) 10:121. doi: 10.1186/s13550-020-00713-2

41. Cunningham SI, Tomasi D, Volkow ND. Structural and functional connectivity of the precuneus and thalamus to the default mode network. Hum Brain Mapp. (2017) 38:938-56. doi: 10.1002/hbm.23429

42. Li R, Utevsky AV, Huettel SA, Braams BR, Peters S, Crone EA, et al. Developmental maturation of the precuneus as a functional core of the default mode network. J Cogn Neurosci. (2019) 31:150619. doi: 10.1162/jocn_a_01426

43. Hermesdorf M, Sundermann B, Feder S, Schwindt W, Minnerup J, Arolt V, et al. Major depressive disorder: findings of reduced homotopic connectivity and investigation of underlying structural mechanisms. Hum Brain Mapp. (2016) 37:1209-17. doi: 10.1002/hbm.23097

44. Li W, Qin W, Liu H, Fan L, Wang J, Jiang T, et al. Subregions of the human superior frontal gyrus and their connections. Neuroimage. (2013) 78:46-58. doi: 10.1016/j.neuroimage.2013.04.011

45. Alagapan S, Lustenberger C, Hadar E, Shin HW, Fröhlich F. Low-frequency direct cortical stimulation of left superior frontal gyrus enhances working memory performance. Neuroimage. (2019) 184:697-706. doi: 10.1016/j.neuroimage.2018.09.064 
46. du Boisgueheneuc F, Levy R, Volle E, Seassau M, Duffau H, Kinkingnehun S, et al. Functions of the left superior frontal gyrus in humans: a lesion study. Brain: a journal of neurology. (2006) 129:3315-28. doi: 10.1093/brain/awl244

47. Xiong G, Dong D, Cheng C, Jiang Y, Sun X, He J, et al. State-independent and -dependent structural alterations in limbic-cortical regions in patients with current and remitted depression. J Affect Disord. (2019) 258:110. doi: 10.1016/j.jad.2019.07.065

48. Lebedeva A, Sundström A, Lindgren L, Stomby A, Aarsland D, Westman E, et al. Longitudinal relationships among depressive symptoms, cortisol, and brain atrophy in the neocortex and the hippocampus. Acta Psychiatr Scand. (2018) 137:491-502. doi: 10.1111/acps.12860

49. Zhang K, Liu Z, Cao X, Yang C, Xu Y, Xu T, et al. Amplitude of low-frequency fluctuations in first-episode, drug-naïve depressive patients: A 5-year retrospective study. PLoS ONE. (2017) 12:e0174564. doi: 10.1371/journal.pone.0174564

50. Bremner JD, Narayan M, Anderson ER, Staib LH, Miller HL, Charney DS. Hippocampal volume reduction in major depression. Am J Psychiatry. (2000) 157:115-8. doi: 10.1176/ajp.157.1.115

51. Korgaonkar MS, Williams LM, Song YJ, Usherwood T, Grieve SM. Diffusion tensor imaging predictors of treatment outcomes in major depressive disorder. Brit J Psychiatry. (2014) 205:321-8. doi: 10.1192/bjp.bp.113. 140376

52. Goldapple K, Segal Z, Garson C, Lau M, Bieling P, Kennedy S, et al. Modulation of cortical-limbic pathways in major depression: treatmentspecific effects of cognitive behavior therapy. Arch Gen Psychiatry. (2004) 61:34-41. doi: 10.1001/archpsyc.61.1.34
53. Song Y, Shen X, Mu X, Mao N, Wang B. A study on BOLD fMRI of the brain basic activities of MDD and the first-degree relatives. Int J Psychiatry Clin Pract. (2020) 24:236-44. doi: 10.1080/13651501.2020.1744663

54. Qiu H, Li X, Luo Q, Li Y, Zhou X, Cao H, et al. Alterations in patients with major depressive disorder before and after electroconvulsive therapy measured by fractional amplitude of low-frequency fluctuations (fALFF). $J$ Affect Disord. (2019) 244:92-9. doi: 10.1016/j.jad.2018.10.099

Conflict of Interest: The authors declare that the research was conducted in the absence of any commercial or financial relationships that could be construed as a potential conflict of interest.

Publisher's Note: All claims expressed in this article are solely those of the authors and do not necessarily represent those of their affiliated organizations, or those of the publisher, the editors and the reviewers. Any product that may be evaluated in this article, or claim that may be made by its manufacturer, is not guaranteed or endorsed by the publisher.

Copyright () 2021 Gao, Wang, Xiong, Ren, Liu, Wei and Li. This is an open-access article distributed under the terms of the Creative Commons Attribution License (CC $B Y)$. The use, distribution or reproduction in other forums is permitted, provided the original author(s) and the copyright owner(s) are credited and that the original publication in this journal is cited, in accordance with accepted academic practice. No use, distribution or reproduction is permitted which does not comply with these terms. 